(C2020, Elsevier. Licensed under the Creative Commons Attribution-NonCommercialNoDerivatives 4.0 International http://creativecommons.org/about/downloads

cc) $(1) \Theta$ 


\title{
Numerical Simulation of Aerodynamic Problems based on Adaptive Mesh Refinement Method
}

\author{
A.V. Struchkov ${ }^{1}$, A.S. Kozelkov ${ }^{1,2,3^{*}}$, K.N. Volkov ${ }^{4}$, \\ A.A. Kurkin ${ }^{2}$, R.N. Zhuckov ${ }^{1}$, A.V. Sarazov ${ }^{1}$ \\ ${ }^{1}$ Federal State Unitary Enterprise "Russian Federal Nuclear Center - All-Russian Research \\ Institute of Experimental Physics" (FSUE "RFNC-VNIIEF”), Nizhny Novgorod Region, Sarov \\ ${ }^{2}$ Federal State-Funded Higher Education Institution "Nizhny Novgorod State Technical \\ University n.a. R.E. Alexeyev" \\ ${ }^{3}$ Sarov Branch Institute of Physics and Technology of State-Funded Independent Higher \\ Education Institution "National Research Nuclear University MEPhI" \\ ${ }^{4}$ Kingston University, London, United Kingdom
}

\begin{abstract}
Numerical simulation of aerodynamic problems using an algorithm, which identifies shock regions and refines the mesh there using an adaptive mesh refinement method, is performed. The static mesh adaptation method is based on cell partitioning by adding new nodes to cell faces. The method of identification of the shock regions using a pressure and density gradient criterion and its verification are presented. The use and applications of the developed algorithm are illustrated, and supersonic and hypersonic flows with strong shock waves are calculated. The results computed explore the shock-wave structure of the flow, which develops near the nose cone section of a body and determines its aerodynamic performance. The aerodynamic performance of bodies with different nose cone designs, including a body with a spike and a pointed cone with an opposing gas jet injected from the nose section, are evaluated based on the proposed method. The method of static mesh adaptation provides a visually sharper picture of the flow around the body and reduces the numerical error of drag coefficient calculations in comparison with wind tunnel measurements. The results computed on meshes of similar resolution constructed with the adaptation method and automatic mesh generator are compared. The results computed on the adaptive mesh are similar to those computed on the mesh generated automatically, but the adaptive mesh has a smaller number of cells.
\end{abstract}

\section{Keywords}

Flight safety; Computational fluid dynamics; Adaptive mesh; Aerodynamics; High-speed flow; Shock wave

*Corresponding author: askozelkov@mail.ru 


\section{Introduction}

Development and implementation of methods and tools that adequately model fundamental physics and allow credible physics-based optimization for future operational high-speed vehicles are becoming more important due to requirements of ensuring their flight safety $[1,2]$. The methods of computational fluid dynamics (CFD) are extensively applied in design and optimization of high-speed vehicles to get more insight into complex flowfields. Computer simulation is particularly attractive due to its relatively low cost and its ability to deliver data that cannot be measured or observed. The quality of CFD calculations of high-speed flows strongly depends on the proper prediction of flow physics. Investigations of heat transfer, skin friction, secondary flows, flow separation and re-attachment effects demand reliable numerical methods, accurate programming and robust working practices.

Demand for high-speed flights and reduced fuel consumption leads to more complex vehicle configurations and engine architectures and consequently creates a need for higher efficiency and less conservative design. This trend leads to a near future where the accuracy of today's state-ofthe-art design tools based on solution of Reynolds-averaged Navier-Stokes equations (RANS) is insufficient. Moreover, important physical processes are poorly represented by RANS-based turbulence models, despite the huge effort devoted to their improvement. In many cases, the limit for what is possible to achieve with standard engineering tools has already been reached, and a step change in methodology is necessary.

In order to make CFD methods feasible for industry design purposes, a number of issues have to be resolved: accuracy (the tools need to be validated based on representative test cases); speed (the analysis time in industry needs to be limited and predictable); robustness (the methods employed in industry need to be reliable and user-friendly); data management capability (due to the large data sets generated, robust routines for data reduction are crucial for efficient industrial implementation); competitiveness (establish best practice guidelines for CFD as an integrated part in an efficient design process).

Industrial simulations are most commonly performed on unstructured meshes because such meshes are more flexible in discretization of domains with complex geometry, and generation of unstructured meshes can be performed automatically [3-6]. However, the main bottleneck of using the unstructured meshes is a possible loss of accuracy because of insufficient mesh resolution near the walls and in shock regions with high gradients of flow variables. 
The construction of a qualitative computational grid is especially important when modelling gas dynamics problems, the solution of which may contain discontinuities (shock waves, contact discontinuities), as well as areas with a rapid change in gas-dynamic variables (rarefaction waves, boundary layers). For the qualitative resolution of such zones, as well as to optimize the calculation time, adaptive meshes are used. Adaptation of the mesh makes it possible to redistribute the source nodes depending on the features of the solution and is part of the process of finding a solution, which allows for most effective calculations taking into account peculiarities of its behaviour.

Adaptive computational meshes are classified according to their functions and construction methods [7]. For example, when solving problems with boundary layers, in which it is necessary to refine the mesh near solid walls, or tasks where the boundaries of the computational domain change their position in space and time, a geometrically adaptive mesh is used [7-9]. The construction of such a mesh is carried out consistently with the geometry of the computational domain. If it is necessary to refine the mesh to the peculiarities of the physical solution of the problem (for example, in the areas of discontinuities, rapid parameter changes), then dynamically adaptive meshes are used. Dynamically adaptive mesh, depending on the method of its construction, can be adaptively embedded or adaptively movable [10-12].

An effective ways to improve the mesh resolution in the region of interest is to use local mesh refinement. This way is often accompanied by adaptive mesh refinements [13-15]. Existing adaptive mesh refinement methods are basically used on structured meshes or on unstructured meshes with a certain shape of cells $[14,15]$.

Algorithm adaptation is necessary for integrating CFD tools in an industrial design environment. In this context, there is a number of aspects to be considered. In order to successfully implement CFD as a natural part of a robust, automated and industrially approved design process chain, aspects such as computational resources, analysis setup and desired engineering output need to be addressed.

This study describes an adaptive mesh refinement method that can be used on arbitrary meshes, and discusses its use for simulations of high-speed flows appearing in aerodynamics. The proposed method automatically identifies regions of local mesh refinement based on the analysis of gradients of physical quantities. The cells belonging to such regions are treated by a refinement 
algorithm, which divides the cells into a number of sub-cells by adding new nodes [13]. The key requirement is that the cells should be convex.

The algorithm demonstrates the best performance as applied to problems with shock waves because shock regions have steep fronts with complex geometrical configurations, and it is rather difficult to resolve them at the stage of mesh generation. The algorithm automatically refines the mesh in the shock region and thus resolves the shock front more accurately. It should also be noted that the proposed algorithm is used independently of the solution process, which means that the computation is first performed on the main mesh, following which the mesh is refined (the static mesh refinement). This does not make the algorithm less valuable, and even in such a form, it provides a considerable gain in accuracy of the final solution. It is demonstrated on the problems of super- and hypersonic flows around different bodies.

To exploit the potential for efficiency improvements achievable by CFD analysis, the numerical tools need to be introduced in an industrial framework. This will increase the level of detail that is possible to represent and quantify accurately in component or module analyses. In order to guarantee accuracy for an industrial application, an extensive validation campaign is planned as part of the project.

\section{Governing equations and numerical method}

In Cartesian coordinates $(x, y, z)$, an unsteady three-dimensional compressible flow is described by the following equation

$$
\frac{\partial W}{\partial t}+\frac{\partial\left(F_{e}-F_{\mu}\right)}{\partial x}+\frac{\partial\left(G_{e}-G_{\mu}\right)}{\partial y}+\frac{\partial\left(E_{e}-E_{\mu}\right)}{\partial z}=H
$$

where $W$ is the vector of the conservative variables, $F_{e}(W), G_{e}(W), E_{e}(W)$ and $F_{\mu}(W, \nabla W), G_{\mu}(W, \nabla W), E_{\mu}(W, \nabla W)$ are the vectors of convective and diffusive fluxes across the surfaces orthogonal to the Cartesian directions, and $H(W, \nabla W)$ is the source term. The vector of conservative variables, flux vectors and source term are given by

$$
W=\left(\begin{array}{c}
\rho \\
\rho u \\
\rho v \\
\rho w \\
\rho E
\end{array}\right), F_{e}=\left(\begin{array}{c}
\rho u \\
\rho u^{2}+p \\
\rho u v \\
\rho u w \\
\rho u H
\end{array}\right), G_{e}=\left(\begin{array}{c}
\rho v \\
\rho u v \\
\rho v^{2}+p \\
\rho v w \\
\rho v H
\end{array}\right), E_{e}=\left(\begin{array}{c}
\rho w \\
\rho u w \\
\rho v w \\
\rho w^{2}+p \\
\rho w H
\end{array}\right), H=\left(\begin{array}{l}
0 \\
0 \\
0 \\
0 \\
0
\end{array}\right),
$$




$$
F_{\mu}=\left(\begin{array}{c}
0 \\
\tau_{x x} \\
\tau_{x y} \\
\tau_{x z} \\
q_{x}+u \tau_{x x}+v \tau_{x y}+w \tau_{x z}
\end{array}\right), G_{\mu}=\left(\begin{array}{c}
0 \\
\tau_{y x} \\
\tau_{y y} \\
\tau_{y z} \\
q_{x}+u \tau_{y x}+v \tau_{y y}+w \tau_{y z}
\end{array}\right), E_{\mu}=\left(\begin{array}{c}
0 \\
\tau_{z x} \\
\tau_{z y} \\
\tau_{z z} \\
q_{x}+u \tau_{z x}+v \tau_{z y}+w \tau_{z z}
\end{array}\right) .
$$

The components of viscous stress tensor and components of heat flux vector are found from the relations

$$
\tau_{i j}=\mu_{e}\left(\frac{\partial v_{i}}{\partial x_{j}}+\frac{\partial v_{j}}{\partial x_{i}}-\frac{2}{3} \frac{\partial v_{k}}{\partial x_{k}} \delta_{i j}\right), \quad q_{i}=-\lambda_{e} \frac{\partial T}{\partial x_{i}} .
$$

Here, $t$ is the time, $\rho$ is the density, $u, v, w$ are the velocity components in the coordinate directions $x, y, z, p$ is the pressure, $T$ is the temperature, $E$ is the total energy per unit mass, and $H$ is the total enthalpy per unit mass. The specific total energy and specific total enthalpy are

$$
E=\varepsilon+\frac{u^{2}+v^{2}+w^{2}}{2}, H=\varepsilon+\frac{p}{\rho}+\frac{u^{2}+v^{2}+w^{2}}{2},
$$

where $\varepsilon$ is specific internal energy. A link between pressure and specific total energy has a form

$$
p=(\gamma-1) \rho\left[\varepsilon-\frac{1}{2}\left(u^{2}+v^{2}+w^{2}\right)\right] .
$$

The Sutherland's law is used to obtain molecular viscosity as a function of temperature

$$
\frac{\mu}{\mu_{*}}=\left(\frac{T}{T_{*}}\right)^{3 / 2} \frac{T_{*}+S_{0}}{T+S_{0}}
$$

where $\mu_{*}=1.68 \cdot 10^{-5} \mathrm{~kg} /(\mathrm{m} \mathrm{s}), T_{*}=273 \mathrm{~K}$ и $S_{0}=110.5 \mathrm{~K}$ for air. Thermal conductivity is expressed in terms of viscosity and Prandtl number, $\lambda=c_{p} \mu / \operatorname{Pr}$. Molecular Prandtl number is fixed at $\operatorname{Pr}=0.72$ for air.

The numerical investigation of phenomena associated with shock wave propagation (e.g. reflection and dispersion) through the use of conservative shock-capturing, high-resolution, Riemann problem-based numerical methods for hyperbolic conservation laws has generated great interest within the fluid dynamics community over recent years. For the computation of unsteady flows, the scheme which is used is of importance. The accuracy of a number of high-resolution schemes to solve complex unsteady viscous flows is evaluated in [19]. 
The solver implemented includes local and global space-time conservation of the solution which limits the diffusion of the solution and loss of precision; high-order scheme for both flow variables and their spatial derivatives for better solution accuracy; novel supersonic shock capturing strategy which does not involve any Riemann solver resulting in less calculation costs.

The non-linear finite volume CFD solver uses implicit scheme. The governing equations are solved with AUSM+ scheme (Advection Upstream Splitting Method) for inviscid fluxes, and central difference scheme for viscous fluxes. The system of finite difference equations is solved with the algebraic multigrid method. The code uses a face centred-based data structure to give the flexibility to run on meshes composed of a variety of cell types.

\section{Mesh adaptation}

The method of mesh adaptation is based on a cell partitioning algorithm, which divides cellgenerating faces [13]. It is considered on a hexahedral cell as an example.

To create new faces, new nodes need to be inserted at the centre of each face and at the midpoint of each edge (Figure 1) of the parent cell.

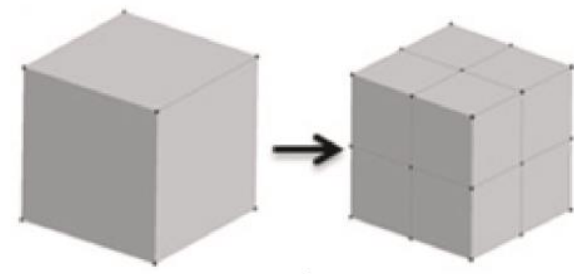

a)

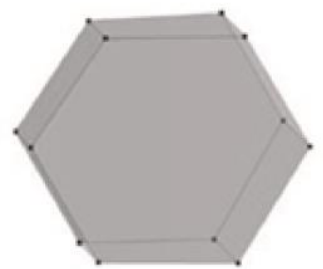

b)

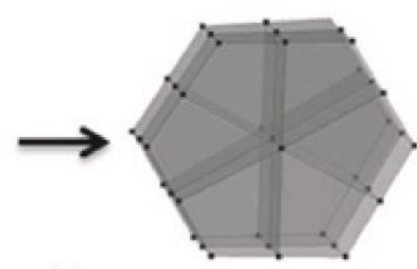

Figure 1. Addition of new nodes at face centres and edge midpoints for hexahedral cell (a) and arbitrary polyhedral cell (b)

To create new faces inside the parent cell, a new node is added at its centre (Figure 2). As a result, one parent cell includes eight level 1 child cells. The algorithm can also be used for arbitrary convex polyhedral cells. 


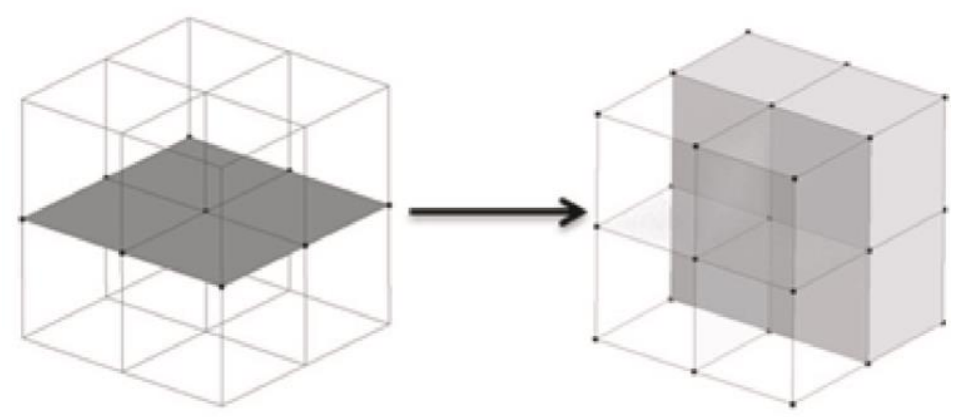

Figure 2. Creation of faces inside a cell and generation of level 1 of child cells

If necessary, the level 1 child mesh can also be processed by the adaptation algorithm in order to produce a level 2 child mesh. The number of further levels is limited only by the minimum cell size, down to which the cell can be divided. Thus, a new adaptive mesh is constructed, which contains locally refined regions and is used for further calculations. Note that the solution calculated on the preceding mesh is used as an initial approximation of the solution when passing on to the child mesh of next level, which saves the time of calculations on the next-level mesh. Before applying the adaptation algorithm, one has to identify the regions, where the mesh needs to be refined, for which purpose adaptation criteria $[16,17]$ are applied.

\section{Mesh adaptation criteria}

One of the most appropriate criteria for the automatic identification of the shock regions produced by transonic and supersonic flows is the gradient of flow quantity $[16,17]$

$$
f=\left(V_{\text {cell }}\right)^{2 / 3} \cdot|\nabla F|
$$

where $V_{\text {cell }}$ is the cell volume, and $\nabla F$ is the gradient of the flow quantity.

The quantity $\left(V_{\text {cell }}{ }^{2 / 3}\right)$ in (5) is used to evaluate the cell's geometric size and to incorporate the dependence of $f$ on the cell volume. Thus, the maximum value of the criterion, in combination with the magnitude of the flow quantity gradient, will be achieved in the cells having the largest volume and a high gradient, which ensures that large cells with significant variations in the flow quantities (like a shock wave) are included in the local refinement region. The minimum value of the criterion $f$ is typical of regions with smooth variations in flow quantities and is achieved in the cells having the smallest volume and a low gradient, as a result of which such cells are excluded from the range of local refinement. 
In this study, as a flow quantity $F$ density or pressure is used. These quantities are the best indicators of shock regions [16], because their gradients in such regions are the highest. A mesh sensitivity analysis is performed using a sequence of three meshes (coarse, intermediate and fine). The results with intermediate mesh are similar to those with fine mesh. The simulations are performed using an intermediate mesh.

\section{Verification and validation}

An example of automatic shock front identification using the criterion (5) in a supersonic flow through a channel with a wedge [18], shown in the Figure 3, is considered. The flow velocity of $697.52 \mathrm{~m} / \mathrm{s}$, corresponding to Mach number of 2, the static pressure of $101325 \mathrm{~Pa}$ and static temperature of $300 \mathrm{~K}$ are specified in the inlet section of the channel.

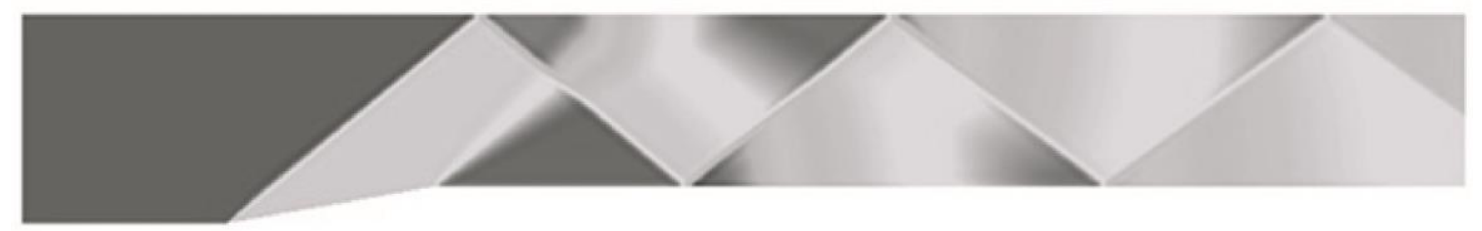

Figure 3. Mach number contours of a supersonic flow in a channel with a wedge

In a supersonic flow in a channel with a wedge, there appears an attached shock wave, which leads to the formation of a shock-wave structure in the channel. Generation and growth of the shock wave, its reflection from the channel walls and interaction with a system of rarefaction waves are observed (region A in the Figure 4). For the purposes of numerical simulations, it is recommended to construct detailed meshes in the regions occupied by such structures to reduce the front smearing (region B in the Figure 4) and increase the accuracy of numerical solution.

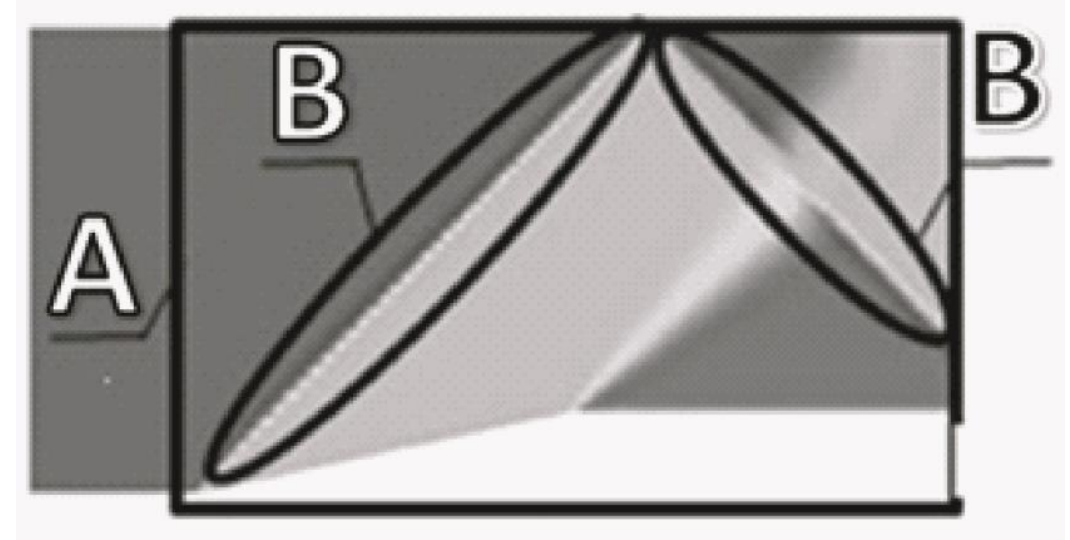

Figure 4. Flow region near the wedge (A) and region of interest (B) in a channel with a wedge 
The field of the criterion $f(1)$ for this case has the form shown in Figure 5.

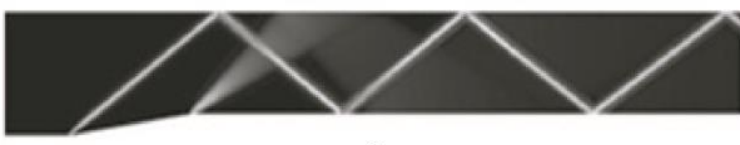

a)

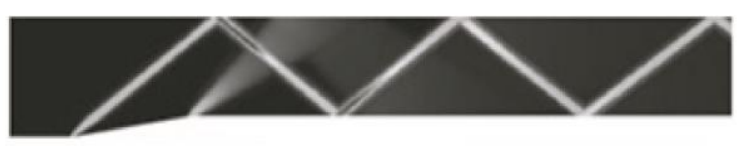

b)

Figure 5. Density gradient (a) and pressure gradient (b) contours in a channel with a wedge

The region of the maximum value of $f(5)$ (depicted using the brightest white colour), for both density and pressure, coincides with the location of the shock front. In this case, the results of using the density- and pressure-based criterion are identical. The region with the maximum value of this criterion is treated as a region for local mesh refinement. Figure 6 shows a level 2 of the mesh generated using the pressure-based criterion.

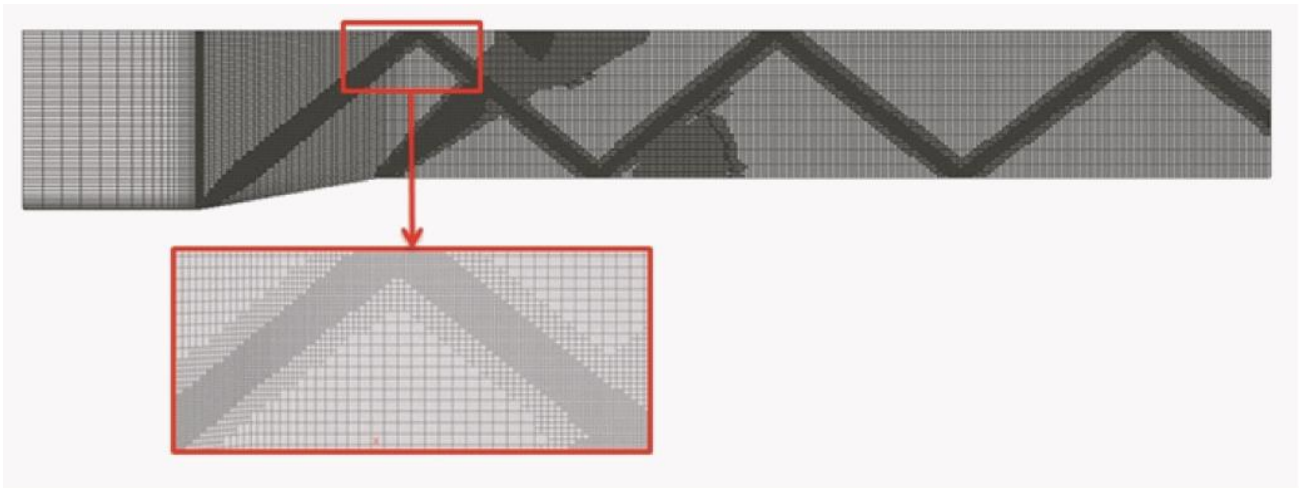

Figure 6. Local mesh refinement (level 2)in a channel with a wedge

To verify the proposed algorithm, the distribution of the total pressure along the channel on the parent mesh is evaluated on the levels 1 and 2 of child meshes, and on a mesh with half step size of the parent mesh spacing (Figure 7). 


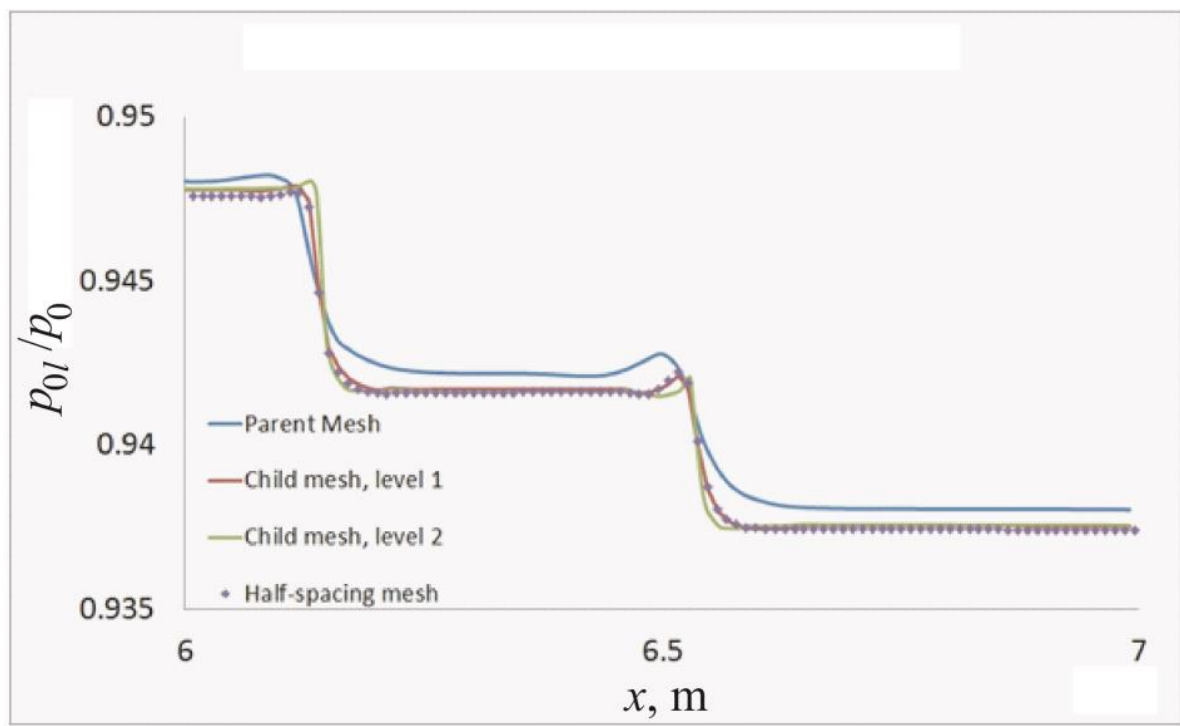

Figure 7. Distribution of normalized total pressure and localization of final shock wave

Figure 7 shows that the shock amplitude in the region of the final series of shock waves is lower on the parent mesh and higher on the child meshes of both adaptation levels and on the half-spacing mesh. Note that the smallest mesh spacing is found in the shock region on the level 2 of child mesh. This refinement, however, only provides a smaller degree of shock front smearing (which, in fact, is reasonable), whereas the shock amplitude remains the same as on the level 1 of child mesh and the half-spacing mesh (results on these meshes are almost identical). Comparison is made for the final series of shock waves, because the initial shock in the channel outlet is weaker there compared to its front part.

These results suggest that the mesh convergence with respect to the shock amplitude is reached on the level 1 of child mesh, and no global refinement is required, as evidenced by the computation on the half-spacing mesh.

Table 1 shows the number of cells of different meshes, and time characteristics of the computations.

Table 1. Number of cells of the meshes and computational time

\begin{tabular}{|c|c|c|c|}
\hline Mesh & $\begin{array}{c}\text { Number of } \\
\text { cells }\end{array}$ & $\begin{array}{c}\text { Time per } \\
\text { iteration, } s\end{array}$ & $\begin{array}{c}\text { Number of } \\
\text { iterations }\end{array}$ \\
\hline Parent & 95,000 & 0.24 & 90 \\
\hline Child mesh, level 1 & 201,204 & 0.54 & 140 \\
\hline Child mesh, level 2 & 563,332 & 1.9 & 190 \\
\hline
\end{tabular}




\begin{tabular}{|l|l|l|l|}
\hline Half-spacing mesh & 760,000 & 2.1 & 140 \\
\hline
\end{tabular}

As noted above, the mesh convergence is reached on the level 1 of child mesh. Considering the reduced time and computational costs due to the use of this mesh, one can conclude that it is preferable to use the mesh adaptation method with local refinement.

\section{Results and discussion}

All the computations reported in this work were performed using the in-house software package LOGOS. LOGOS is an engineering analysis software intended for three-dimensional conjugate convective heat and mass transfer, aerodynamics and hydrodynamics simulations on parallel computers. LOGOS has been successfully verified and demonstrated its sufficiently high utility as applied to a series of different hydrodynamic tests [5, 19-21], including turbulent and unsteady flow $[22,23]$ and geophysical processes [24-26].

\subsection{Hypersonic flow around a body with a spike}

According to [27-29], projecting components on the aircraft forebody are used to reduce the aerodynamic drag. This excites interest of engineers, who develop supersonic aerospace vehicles, to such forebody designs. Such a forebody design can be implemented as a sharp projecting spike. The presence of a spike in combination with a streamlined body reduces the drag in the supersonic phase of the flight because deceleration occurs in a single detached shock wave rather than in a series of oblique shocks [27-29]. The drag is also reduced if the spike is mounted in front of a blunt body. The spike enables more efficient use of the thrust of high-speed aircraft propulsion systems and considerably reduces thermal fluxes by creating a zone of recirculating flow, the size of which is determined by the spike design. The spike mounted on the forebody gives advantages during both take-off and flight [30-32]. The flow structure near a body with a spike is shown in the Figure 8. 


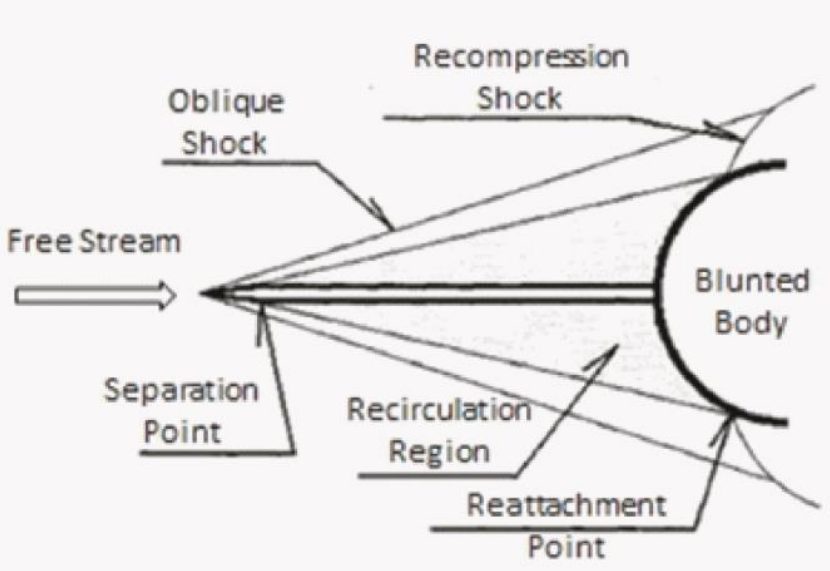

a)

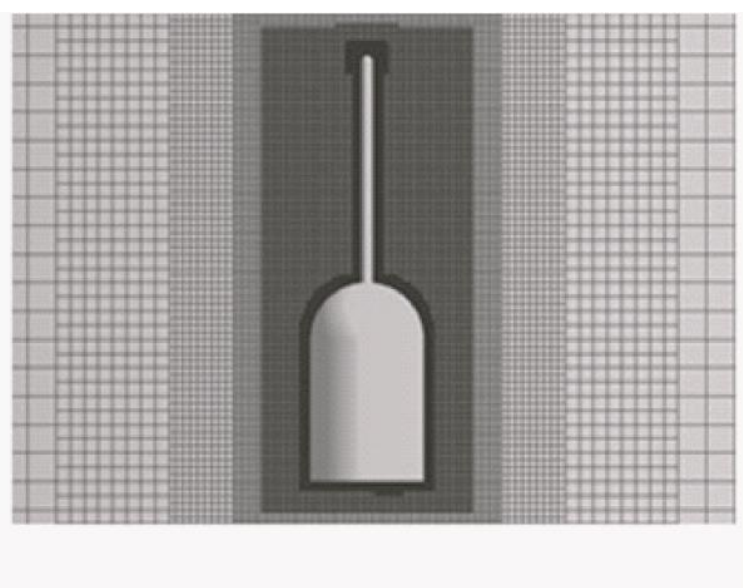

b)

Figure 8. Flow structure near a body with a spike (left) and computational model (right)

The influence of the mesh adaptation algorithm on the calculated drag coefficient is considered and the computed results are compared with experimental data [30-32]. The number of cells of the unstructured mesh (Figure 8) is about 10 million. Local mesh refinement of the discrete model is specified in the anticipated shock region. To calculate the flow around the body, oncoming flow has a velocity of $886.425 \mathrm{~m} / \mathrm{s}$ (Mach number is about 6), static temperature is $54.87 \mathrm{~K}$, static pressure is $525.67 \mathrm{~Pa}$. Angles of attack are fixed at 1,2 and 4 degrees.

A computation on the parent mesh is performed for each angle of attack. Then, a local mesh refinement in the regions of the oblique and detached shock waves is introduced. Figure 9 shows that the direction of the detached shock changes depending on the angle of attack. The use of the mesh adaptation method in conjunction with the Mach-based criterion makes it possible to construct an adaptive mesh (Figure 10) with a local refinement region corresponding to the path of the propagating shock front. Thus, the mesh adaptation method allows obtaining three different adaptive meshes based on the parent mesh that contain local mesh refinement regions matched with the front of the shock wave developing under varied given conditions. 


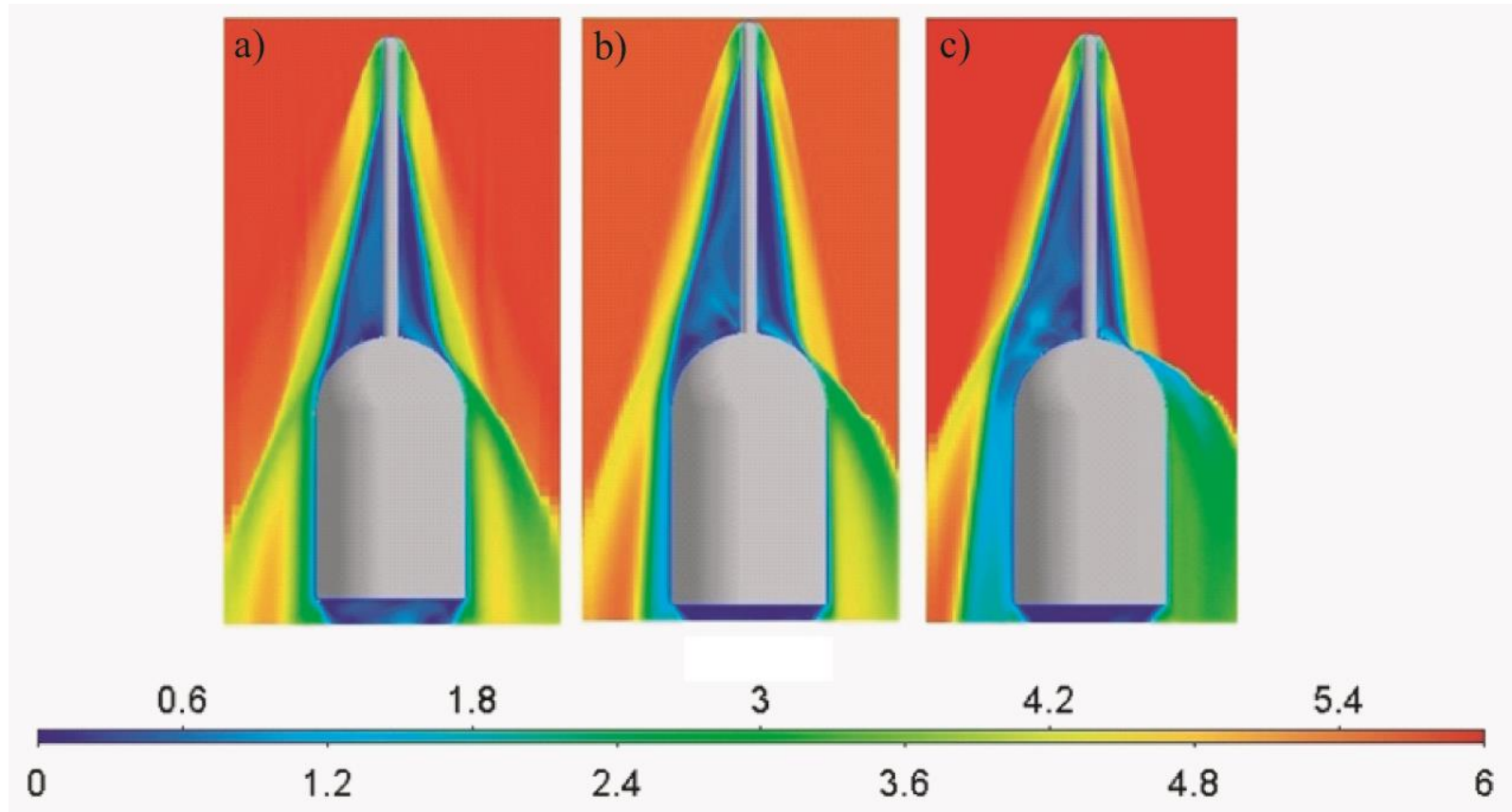

Figure 9. Mach number contours for angle of attach of 1 (a), 2 (b) and 4 (c) degrees

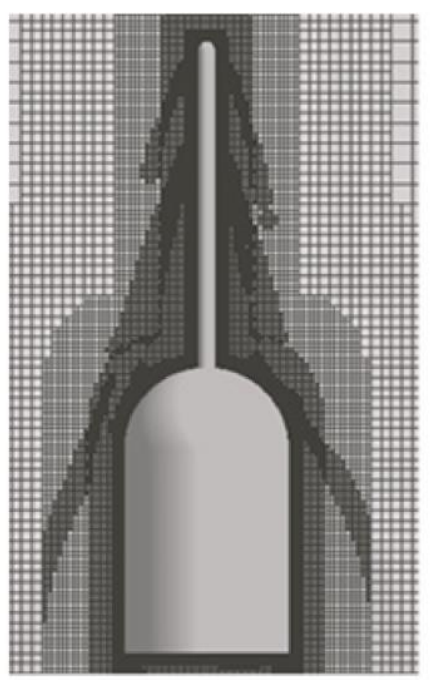

a)

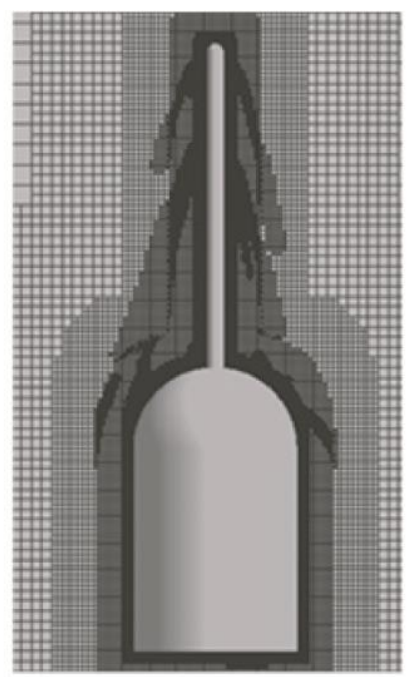

b)

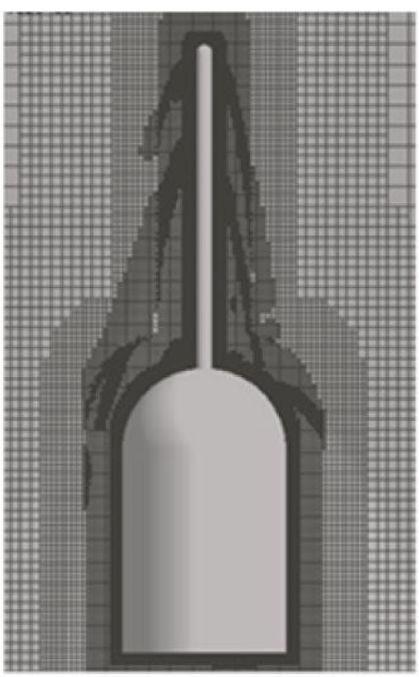

c)

Figure 10. Adaptive mesh refinement for angle of attach of 1 (a), 2 (b) and 4 (c) degrees

As a result of the computation, the drag coefficient is found (Table 2). Dependence of the drag coefficient on angle of attack is presented in the Figure 11.

Table 2. Drag coefficient of a body with a spike

\begin{tabular}{|c|c|c|c|c|c|}
\hline $\begin{array}{c}\text { Angle of } \\
\text { attack, deg }\end{array}$ & Experiment & \multicolumn{2}{|c|}{$\begin{array}{c}\text { Computation on parent } \\
\text { mesh }\end{array}$} & $\begin{array}{c}\text { Computation on level 1 } \\
\text { of child mesh }\end{array}$ \\
\cline { 3 - 6 } & & Drag & Error, \% & Drag & Error, \% \\
\hline
\end{tabular}




\begin{tabular}{|l|l|l|l|l|l|}
\hline 1 & 0.242 & 0.263 & 8.9 & 0.244 & 1.00 \\
\hline 2 & 0.293 & 0.316 & 8.0 & 0.293 & 0.13 \\
\hline 4 & 0.460 & 0.497 & 8.1 & 0.462 & 0.50 \\
\hline
\end{tabular}

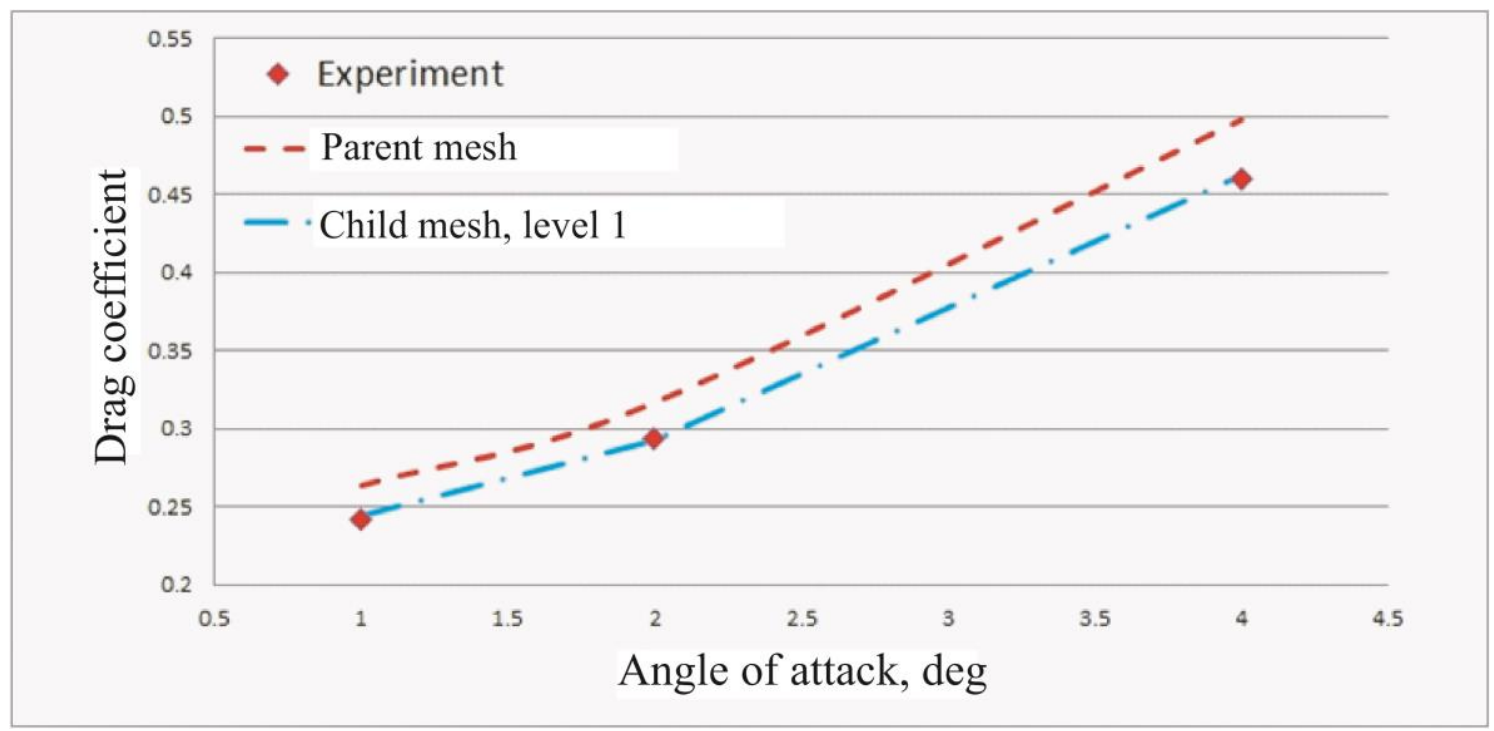

Figure 11. Dependences of drag coefficient on angle of attack

The resulting values are compared with experimental data. The data shown in the Table 1 and Figure 11 point at qualitative improvement in the resulting values of the drag coefficient due to the mesh adaptation method, because it has enabled more accurate resolution of the developing shock waves. Table 3 shows the size of the mesh cells used in the computations.

Table3. Mesh size

\begin{tabular}{|c|c|c|}
\hline $\begin{array}{c}\text { Angle of } \\
\text { attack, deg }\end{array}$ & $\begin{array}{c}\text { Number of cells, } \\
\text { parent mesh }\end{array}$ & $\begin{array}{c}\text { Number of cells, } \\
\text { level } 1 \text { of child mesh }\end{array}$ \\
\hline 1 & $3,552,199$ & $5,983,175$ \\
\hline 2 & $3,552,199$ & $5,431,852$ \\
\hline 4 & $3,552,199$ & $5,553,149$ \\
\hline
\end{tabular}

In the static mesh adaptation method, the number of cells in the child mesh increases compared to the parent mesh. It is established by practical analysis that a mesh comparable in resolution to the level 1 of child mesh contains over 10 million cells. It takes much more time to obtain a numerical solution on a mesh of this size. This is indicative of an undeniable advantage of the adaptive mesh in that it contains a smaller number of cells, which correlates directly with the time taken by the computation. 


\subsection{Supersonic flow around a cone with gas injection}

According to [33-36], another practical drag-reducing method for vehicles flying in the dragproducing atmosphere is injection of an opposing jet into the oncoming air flow. This method is also used to reduce thermal heating of the forebody by creating a zone of cold recirculation near it [33-36]. The stronger is the injected jet, the lower is the drag, because the zone of low-pressure recirculation grows and the detached shock, which is the main cause of the drag, gets weaker. Figure 12 shows the flow structure forming near a blunt forebody. In general, the forebody can also be pointed, and the flow structure in this case will be the same.

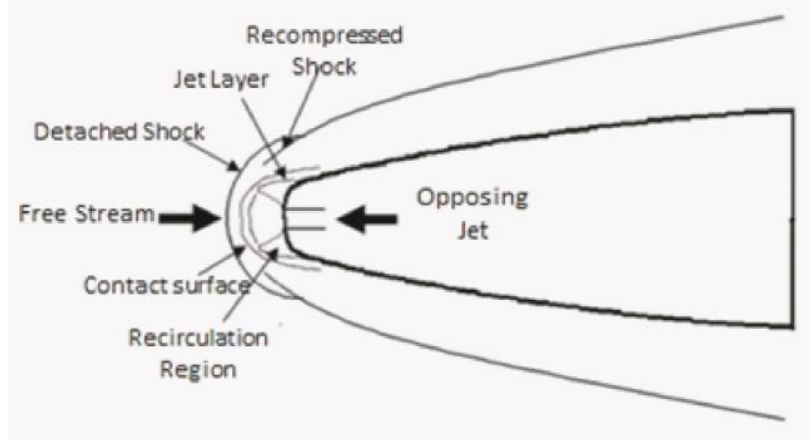

a)

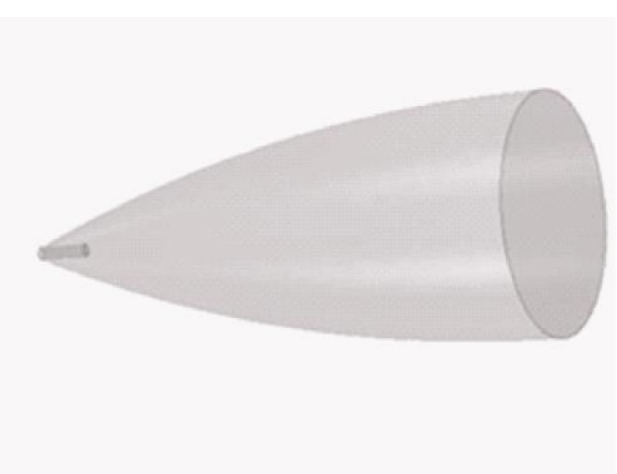

b)

Figure 12. Flow structure near a body (a) and computational model (b)

The influence of the mesh adaptation algorithm on the calculated drag coefficient for the case of a supersonic flow around a pointed model is considered and the computed results are compared with experimental data [34]. The domain has a central axial symmetry and a cone-shaped body having a diameter of $40 \mathrm{~mm}$ and length of $85 \mathrm{~mm}$ is used in calculations. The unstructured parent mesh shown in the Figure 13 contains about 732,000 cells.

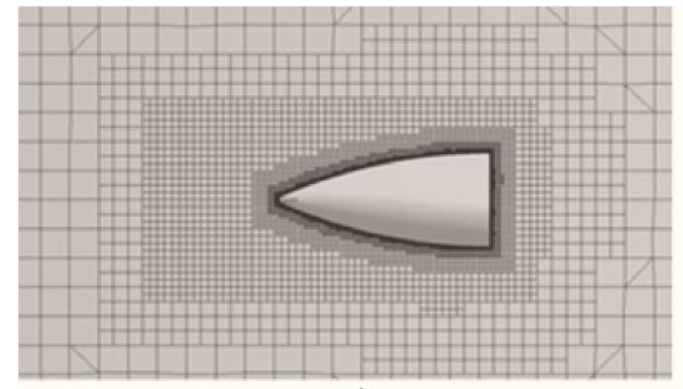

a)

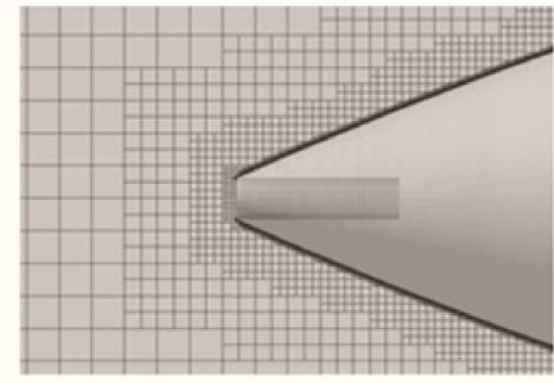

b)

Figure 13. General view of the mesh (a) and mesh near the forebody (b) 
At the mesh generation stage, a general range of local refinement near the body without specifying the higher-resolution shock regions is defined. The flow around the body is calculated for an oncoming flow of Mach number of 3.98 with a static temperature of $96.9 \mathrm{~K}$ and pressure of 9334.7 $\mathrm{Pa}$ at the outer boundary. The angle of attack is fixed at 0 degrees.

A flow of nitrogen with Mach number of 1 , total temperature $300^{\circ} \mathrm{K}$, and pressure $729,028.867$ $\mathrm{Pa}$ is injected into the oncoming flow through a 1-mm-radius round hole in the model forebody.

Figure 14 shows the Mach number field near the surface of the whole body and around its forebody. The parent mesh does not provide sufficient resolution of developing shocks, and the flow structure is smeared. The insufficient mesh resolution is also evidenced by the resulting value of the drag coefficient, which equals 0.046 and differs from the experimental data by 17 percent.
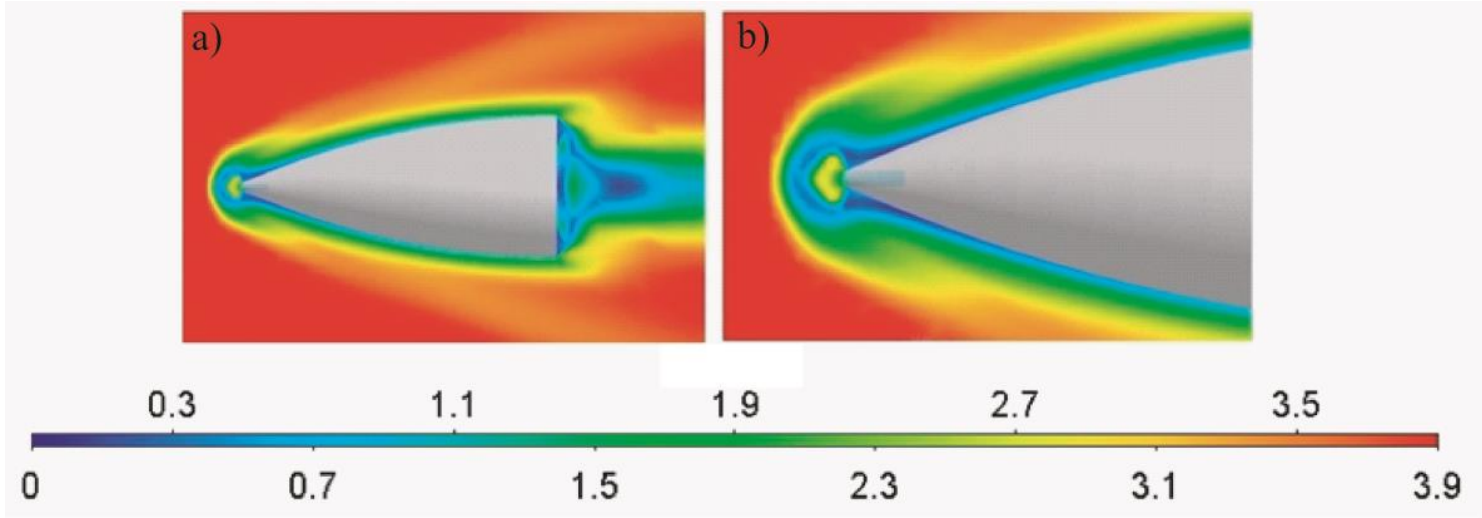

Figure 14. Mach number contours on parent mesh around the body (a) and near its forebody (b)

After its treatment by the mesh adaptation method, the mesh has local refinement regions along the shock front. It takes a total of three adaptation steps to obtain the desired accuracy of the solution (about 10 percent difference from experimental data). The Mach number field for the level 3of child mesh is shown in the Figure 15. 

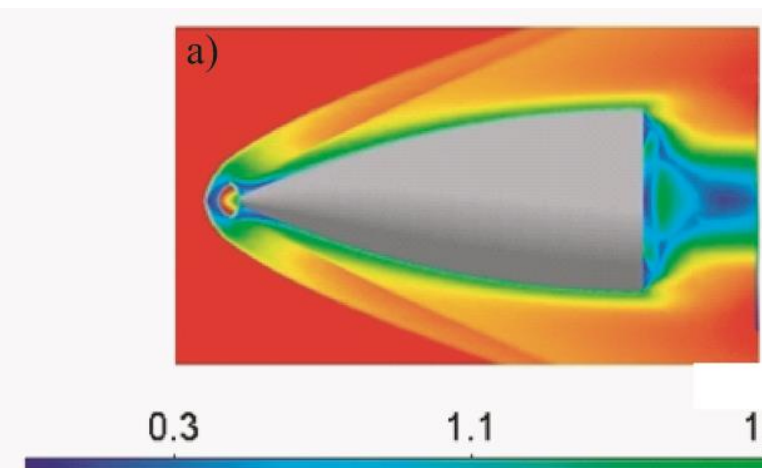

0 0.7

1.1

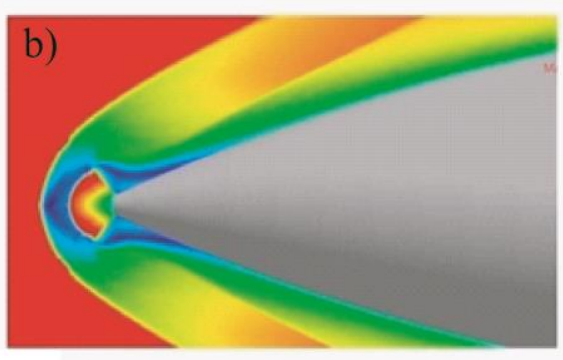
1.9

2.7

2.3

3.5

1.5

$2.3 \quad 3.1$

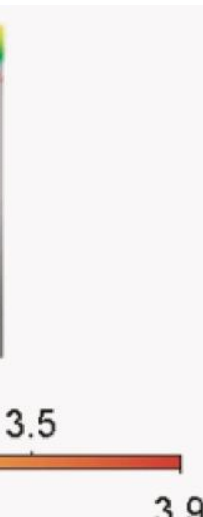

Figure 15. Mach number contours on level 3of child mesh around the body (a) and near its forebody (b)

Figure 15 shows that the level 3 of child mesh provides a high-quality flow structure representation near the body. The front of the detached shock and the region of low-pressure recirculation are clearly visible. As a consequence of the better flow structure resolution, the difference between the resulting drag coefficient and the experimental value becomes as small as 10.2 percent. Child meshes of all adaptation levels are shown in the Figure 16. The meshes are refined locally in the regions, where the shock occurs. As a result of partitioning, parent cells are divided into arbitrary polyhedrons.

a)
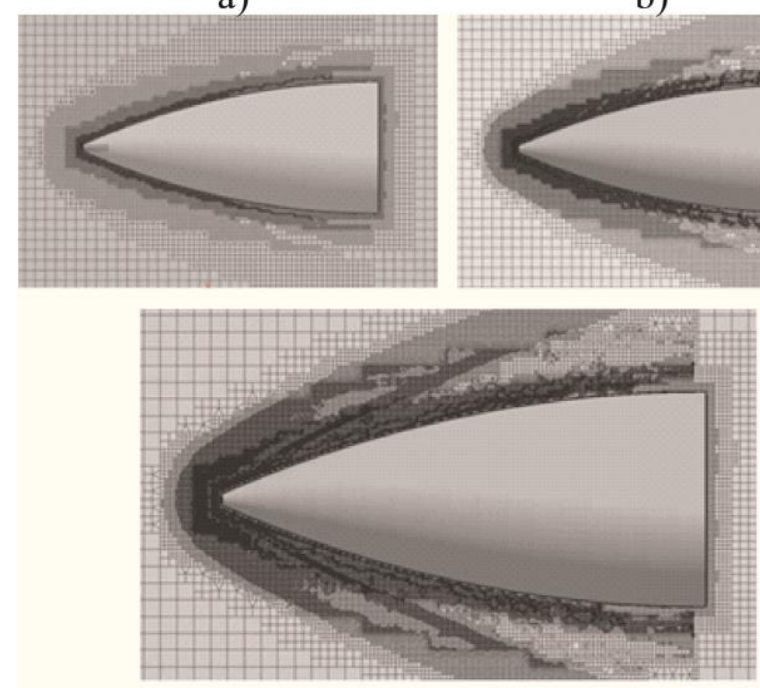

c)

b)

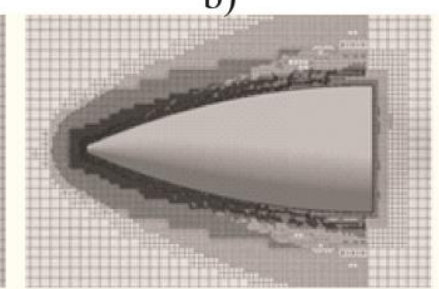

Figure 16. Child meshes of levels 1 (a), 2 (b) and 3(c) near the body

To assess the efficiency of the adaptation method, a mesh comparable in resolution to the level 2 of child mesh is constructed. For this purpose, a cone-shaped local refinement block is inserted 
around the body, the mesh spacing in which is determined by the mesh spacing of the level 2 of child mesh. The resulting mesh is shown in the Figure 17. It contains about 10.2 millions of cells.

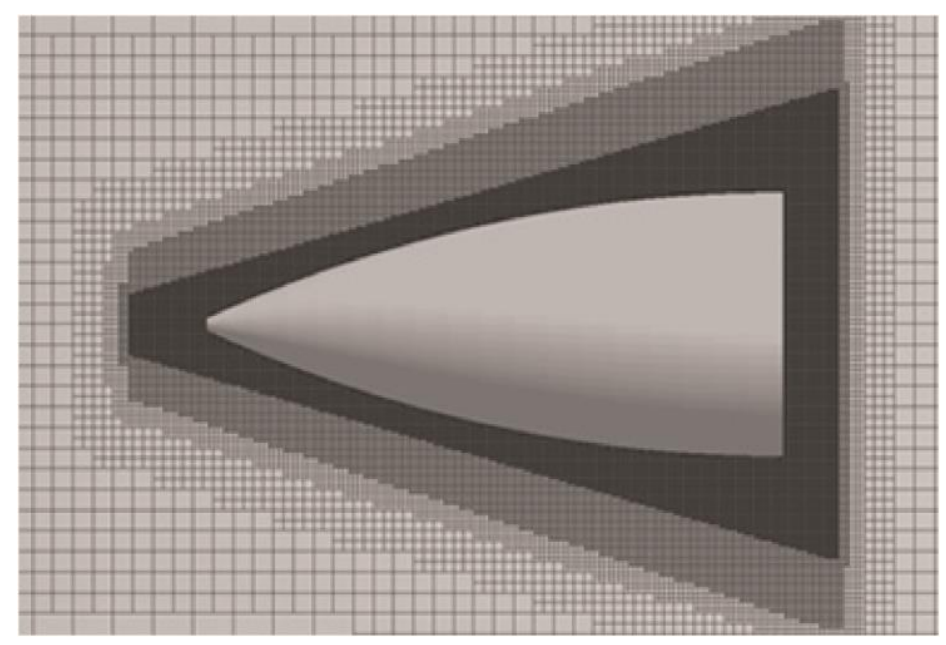

Figure 17. Mesh near the body

This mesh is used to perform a computation, the results of which are shown in the Table4 in the form of the calculated drag coefficient.

Table4. Drag coefficient of a cone with gas injection

\begin{tabular}{|c|c|c|}
\hline Mesh & Drag coefficient & Error, \% \\
\hline Parent mesh & 0.0463 & 17.2 \\
\hline Level 1 of child mesh & 0.0477 & 14.7 \\
\hline Level 2 of child mesh & 0.0484 & 13.4 \\
\hline Level 3 of child mesh & 0.0502 & 10.2 \\
\hline Fine mesh & 0.0485 & 13.7 \\
\hline Experiment & 0.0560 & - \\
\hline
\end{tabular}

One can conclude that the error in the calculated drag coefficient decreases with each adaptation step. In addition, the fine mesh and the level 2of child mesh are comparable in their error, but the fine mesh contains one and a half times as many cells. If a mesh comparable in resolution to the level 3 of child mesh is considered, then it contains about 53 million cells, which makes the computation much more expensive, whereas the level 3 of child mesh has as little as 13 million of cells (Table 5). 
Table5. Mesh size

\begin{tabular}{|c|c|}
\hline Mesh & Number of cells \\
\hline Parent mesh & 731,116 \\
\hline Child mesh, level 1 & $1,330,944$ \\
\hline Child mesh, level 2 & $5,912,204$ \\
\hline Child mesh, level 3 & $12,861,227$ \\
\hline Fine mesh & $8,923,102$ \\
\hline
\end{tabular}

It is fair to note that the cell count of the mesh created by the mesh generator using the local refinement blocks directly depends on the competence of the engineer. The blocks are formed as primitive shapes (cone, sphere, cylinder, parallelepiped), and it is hard to resolve complexgeometry shock fronts if there is no local refinement in the region outside the front. That is why the number of cells in such meshes is quite large. This circumstance confirms the advantage of the adaptive meshes.

\section{Conclusion}

Accurate aerodynamic prediction is critical for the design and optimization of hypersonic vehicles. High-speed compressible flows involve important and interesting fluid phenomena typically exhibiting shock waves, rarefactions and related complexities. Because of these difficulties in obtaining validation data for hypersonic flows, designers are forced to rely heavily on computational fluid dynamics. The accuracy of today's industrial CFD tools is insufficient to be able to produce reliable predictions for aerospace applications. This work was undertaken as part of a larger effort to establish a common CFD code for aerodynamic applications, and involves some basic validation studies. Nevertheless the results given here provide useful information and some confidence that turbulence models acceptable to high-speed aerodynamics and compressible flows can be identified with comparable performance to models already used in these areas.

The possibility of using the method of static mesh adaptation in simulations of super- and hypersonic flow around various bodies characterized by the formation of a bow shock is considered. The proposed algorithm enables automatic local mesh refinement in the shock region, which improves the accuracy of the resulting solution. The developed techniques are applied to the problems of high-speed aerodynamics. In general, use of the adaptive meshes reduces the discrepancy between computational and experimental data by 7 percent. As a result of using this 
method, the cell count increases, but it is still smaller than in the case of global mesh refinement, which proves the efficiency of the proposed algorithm as applied to this class of problems.

\section{Acknowledgments}

This work has been funded by grants of the President of the Russian Federation for state support of research projects by young doctors of science (MD-4874.2018.9) and state support of leading schools of thought of the Russian Federation (NSh-2685.2018.5).

\section{References}

1. Smirnov N.N. Ensuring safety of space flights. Acta Astronautica, 2017, 135, 1-5.

2. Silnikov M.V., Guk I.V., Nechunaev A.F., Smirnov N.N. Numerical simulation of hypervelocity impact problem for spacecraft shielding elements. Acta Astronautica, 2018, 150, 56-62.

3. Yu.N. Deryugin, R.N. Zhuchkov, D.K. Zelenskiy, A.S. Kozelkov, A.V. Sarazov, N.F. Kudimov, Yu.M. Lipnickiy, A.V. Panasenko, A.V. Safronov, Validation results for the LOGOS multifunction software package in solving problems of aerodynamics and gas dynamics for the lift-off and injection of launch vehicles, Mathematical Models and Computer Simulations, 7(2) (2015) 144-153.

4. G. Jothiprasad, D.J. Mavriplis, D.A. Caughey, Higher-order time integration schemes for the unsteady Navier-Stokes equations on unstructured meshes, Journal of Computational Physics 191(2) (2003)542-566.

5. A.S. Kozelkov, V.V. Kurulin, S.V. Lashkin, R.M. Shagaliev, A.V. Yalozo, Investigation of supercomputer capabilities for the scalable numerical simulation of computational fluid dynamics problems in industrial applications, Computational Mathematics and Mathematical Physics 56(8) (2016) 1506-1516.

6. D.J. Mavriplis, Unstructured mesh discretizations and solvers for computational aerodynamics, AIAA Paper2007-3955.

7. W.D. Henshaw, Adaptive mesh refinement on overlapping grids, Lecture Notes in Computational Science and Engineering 35 (2005) 59-71.

8. K. Ito, T. Kunugi, H. Ohshima, A high-precision unstructured adaptive mesh technique for gasliquid two-phase flows, International Journal for Numerical Methods in Fluids 67(11) (2011) 1571-1589.

9. O. Antepara, O. Lehmkuhl, R. Borrell, J. Chiva, A. Oliva, Parallel adaptive mesh refinement for large-eddy simulations of turbulent flows, Computers and Fluids 110 (2015) 48-61.

10. K. Shi, S. Fu, Study of shock/blade tip leakage vortex/boundary layer interaction in a transonic rotor with IDDES method, ASME Paper GT2013-95252. 
11. X. Su, Accurate and robust adaptive mesh refinement for aerodynamic simulation with multiblock structured curvilinear mesh, International Journal for Numerical Methods in Fluids 12(77) (2015) 747-766.

12. J. Gou, X. Yuan, X. Su, Adaptive mesh refinement method based investigation of the interaction between shock wave, boundary layer, and tip vortex in a transonic compressor, Proceedings of the Institution of Mechanical Engineers, Part D: Journal of Aerospace Engineering 232(4) (2018) 694-715.

13. A.V. Struchkov, A.S. Kozelkov, R.N. Zhuchkov, A.A. Utkina, A.V. Sarazov, Numerical simulations of aerodynamic problems with solution-specific static mesh adaptation, Mathematical Simulation of Physical Processes 2 (2019)55-67.

14. P.A. Zegeling, Theory and application of adaptive moving grid methods / Adaptive Computations: Theory and Algorithms. Beijing, Science Press, 2007,279-332.

15. A. Harten, High resolution schemes for hyperbolic conservation laws, Journal of Computational Physics 49(3) (1983) 357-393.

16. J.F. Daunenhofer, J.R. Baron, Grid adaption for the 2D Euler equations. AIAA Paper 85-0484. 17. G.P. Warren, W.K. Andersom, J.L. Thomas, S.L. Krist, Grid convergence for adaptive methods, AIAA Paper 91-1592.

18. K. Volkov, Multigrid and preconditioning techniques in CFD applications / CFD Techniques and Thermo-Mechanics Applications. Springer International Publishing, 2018, 83-149.

19. P. Bulat P., K. Volkov, Simulation of shock wave interaction and reflection phenomena in compressible supersonic flows, Advances in Chemistry Research 49 (2019) 169-216.

20. A.S. Kozelkov, A.A. Kurkin, M.A. Legchanov, V.V. Kurulin, E.S. Tyatyushkina, Y.A. Tsibereva, Investigation of the application of RANS turbulence models to the calculation of nonisothermal low-Prandtl-number flows, Fluid Dynamics 50(4) (2015) 501-513.

21. V.B. Betelin, R.M. Shagaliev, S.V. Aksenov, I.M. Belyakov, Yu.N. Deryuguin, A.S. Kozelkov, D.A. Korchazhkin, V.F. Nikitin, A.V. Sarazov, D.K. Zelenskiy, Mathematical simulation of hydrogen-oxygen combustion in rocket engines using LOGOS code, Acta Astronautica 96 (2014) 53-64.

22. A.S. Kozelkov, O.L. Krutyakova, A.A. Kurkin, V.V. Kurulin, E.S. Tyatyushkina, Zonal RANS-LES approach based on an algebraic Reynolds stress model, Fluid Dynamics50(5) (2015) $621-628$.

23. A.S. Kozelkov, V.V. Kurulin, Eddy resolving numerical scheme for simulation of turbulent incompressible flows, Computational Mathematics and Mathematical Physics55(5) (2015) 12551266. 
24. A.S. Kozelkov, A.A. Kurkin, E.N. Pelinovsky, E.S. Tyatyushkina, V.V. Kurulin, N.V. Tarasova, Landslide-type tsunami modeling based on the Navier-Stokes Equations, Science of Tsunami Hazards:The International Journal of the Tsunami Society 35(3) (2016) 106-144.

25. A.S. Kozelkov, A.A. Kurkin, E.N. Pelinovsky, V.V. Kurulin, E.S. Tyatyushkina, Numerical modeling of the 2013 meteorite entry in Lake Chebarkul, Russia, Natural Hazards and Earth System Sciences 17 (2017) 671-683.

26. A.S. Kozelkov, A.A. Kurkin, V.V. Kurulin, S.V. Lashkin, N.V. Tarasova, E.S. Tyatyushkina, Numerical modeling of the free rise of an air bubble, Fluid Dynamics 51(6) (2016) 709-721.

27. R. Kalimuthu, R.C. Mehta, E. Rathakrishnan, Drag reduction for spike attached to blunt-nosed body at Mach 6, Journal of Spacecraft and Rockets47(1) (2010)219-222.

28. J.P. Reding, R.A. Guenther, B.J. Ritchter, Unsteady aerodynamic considerations in the design of a drag-reduction spike, Journal of Spacecraft and Rockets14(1) (1977) 54-60.

29. K. Koeing, D.H. Bridges, G.T. Chapman, Transonic flow modes of an axisymmetric blunt body, AIAA Journal27(9) (1989) 1301-1302.

30. N. Motoyama, K. Mihara, R. Miyajima, T. Watanuki, H. Kubota, Thermal protection and drag reduction with use of spike in hypersonic flow, AIAA Paper2001-1828.

31. R. Kalimuthu, Experimental investigation of hemispherical nosed cylinder with and without spike in a hypersonic flow. PhD Thesis, Department of Aerospace Engineering, Indian Institute of Technology, Kanpur, India, April 2009.

32. R.C. Mehta, Flowfield computations over conical, disc and flat spiked body at Mach 6, AIAA Paper 2009-03259.

33. I. Tamada, S. Aso, Y. Tani, Numerical study of the effect of the opposing jet on reduction of aerodynamic heating with different nose configurations, Proceedings of the 26th Congress of International Council of the Aeronautical Sciences, 14-19 September 2008, Anchorage, USA. 2008, 4, 1898-1908.

34. K. Hayashi, S. Aso, Y. Tani, Numerical study of thermal protection system by opposing jet, AIAA Paper 2005-188.

35. K. Hayashi, S. Aso, Y. Tani, Experimental study of thermal protection system by opposing jet in supersonic flow, Journal of Spacecraft and Rockets43(1) (2006) 233-238.

36. G. Cheng, K. Neroorkar, Y. Chen, T. Wang, E. Daso, Numerical study of flow augmented thermal management for entry and re-entry environments, AIAA Paper 2007-4560. 